\title{
Bimodal Force Spectroscopy as a Technique to Determine the Young's Moduli of Protein Fibrils and Nanoparticles
}

\author{
Owen Griffith Jones ${ }^{1}$ and Jay Gilbert ${ }^{1}$
}

1. Department of Food Science, Purdue University, West Lafayette, Indiana, USA.

Colloidal protein structures have many applications in foods and packaging, some of which are dependent upon the mechanical attributes of the colloidal structures. Deformability of colloidal materials defines their capacity to operate as emulsifiers [1] or to control the rheological properties in concentrated suspensions [2]. There is then a strong interest to better understand the mechanical properties of individual colloidal structures. However, obtaining a reliable and quantitative estimate of these properties remains a challenge.

Atomic force microscopy (AFM) physically probes the surfaces of samples by observing the flexure of a small cantilever as it interacts with the surface, providing detail of both topography and mechanics across a 2-dimensional window [3]. More detail on the various static or dynamic AFM techniques to obtain mechanical information of biological samples, including traditional force spectroscopy, force mapping, contact resonance modes, or peak force modulation, can be found in the literature [4]. One common limitation in these techniques is that many operate as point-by-point measurements and are correspondingly slow with low spatial resolution. One technique that can overcome these limitation is bimodal force spectroscopy (BFS), which is a multi-frequency dynamic AFM technique wherein a single cantilever is made to simultaneously oscillate at a low and high frequency with constant amplitude and constant frequency, respectively [5]. While topographical features are tracked in the lowfrequency oscillation, differences in the mechanical properties of the sample are obtained from relative changes in the frequency within the high frequency channel [5].

To verify a direct relation between frequency shifts during BFS operation and the Young's modulus of polymeric materials, three polymer films were prepared and tested using BFS. Poly(ethylene), poly(caprolactone), and poly(styrene) were mold-casted as films, and their resistance to extensional stress was determined using a mechanical testing apparatus. Polymer films were then measured by BFS, and the frequency shift of the higher eigenmode was found to correlate linearly with the relative quantity of extensional Young's modulus from bulk testing (Fig. 1). This validated the operation of BFS for measurement of elastic properties among surfaces of polymeric materials.

Protein fibrils and nanoparticles are two colloidal structures of interest for development of higher-order application in foods or packaging materials. Protein fibrils are assembled from peptides or whole proteins by coordinated hydrogen bonds, forming very stiff fibrous structures that are micrometers in length but only 5-20 nanometers in width [6]. $\beta$-lactoglobulin, in particular, forms fibrils during prolonged heat treatment that have been utilized to create gels and films [7]. Nanoparticles, on the other hand, may be formed from poorly water-soluble proteins, such as the maize-based protein zein, by sudden precipitation from non-aqueous solutions. Zein nanoparticles possess diameters of $\sim 100-500$ nanometers and have been investigated as controlled delivery vehicles or as emulsion stabilizers [8].

Protein fibrils and zein nanoparticles were prepared and deposited on mica or polymeric films for characterization of their mechanical properties using BFS. BFS was able to obtain high-resolution 
topographical detail of both colloidal structures, as well as consistent frequency shifts of the higher eigenmode of the cantilevers. By considering the frequency shift of the cantilever during measurement of the known polymer films as a calibration (Fig. 1), Young's moduli of the protein fibrils and zein nanoparticles were determined to be $1.6 \mathrm{GPa}$ and $850 \mathrm{MPa}$, respectively, which are consistent for the materials. Overlays of the determined Young's moduli with topographical detail yielded maps describing the relation between structural components and mechanical properties (Fig. 2).

In order to identify potential changes in the mechanical properties of protein nanostructures within composite materials, BFS was used to characterize zein nanoparticles and protein fibrils in modified cellulose films. Results indicated that both the Young's modulus of both nanostructures were unchanged by their placement in the composites. However, there were changes noted in the regions surrounding the nanostructures, which will be discussed as a potential implication of the relative density of matrix material in these regions [9].

\section{References:}

[1] B Brugger, J Vermant, W Richtering, Physical Chemistry Chemical Physics 12, (2010), p.14573.

[2] J Mattsson et al, Nature 462 (2009), p. 83.

[3] H-J Butt, B Cappella, M Kappl, Surface Science Reports 59 (2005), p. 1.

[4] OG Jones, Current Opinion in Food Science 9 (2016), p. 77.

[5] R Garcia, R Proksch, European Polymer Journal 49 (2013), p. 1897.

[6] OG Jones, R Mezzenga, Soft Matter 8 (2012), p. 876.

[7] TPJ Knowles, et al., Nature Nanotechnology 5 (2010), p. 204.

[8] CJ Cheng, OG Jones, Food Hydrocolloids 69 (2017), p. 28.

[9] The authors acknowledge funding from United States Department of Agriculture Hatch Act.

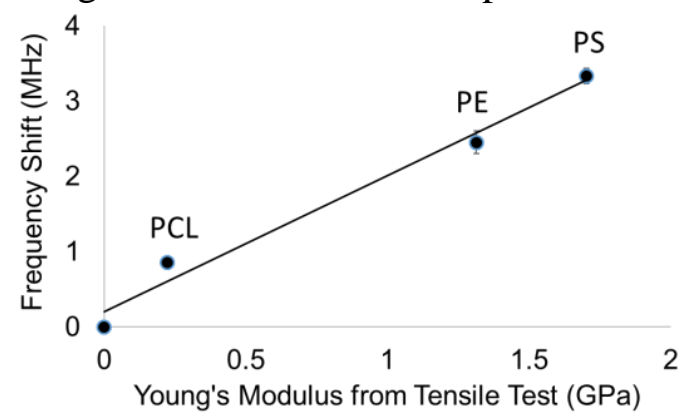

Figure 1. Shift in the oscillation frequency of the cantilever's second eigenmode during contact with PCL, PE, or PS films in relation to the Young's modulus from bulk tensile measurements.
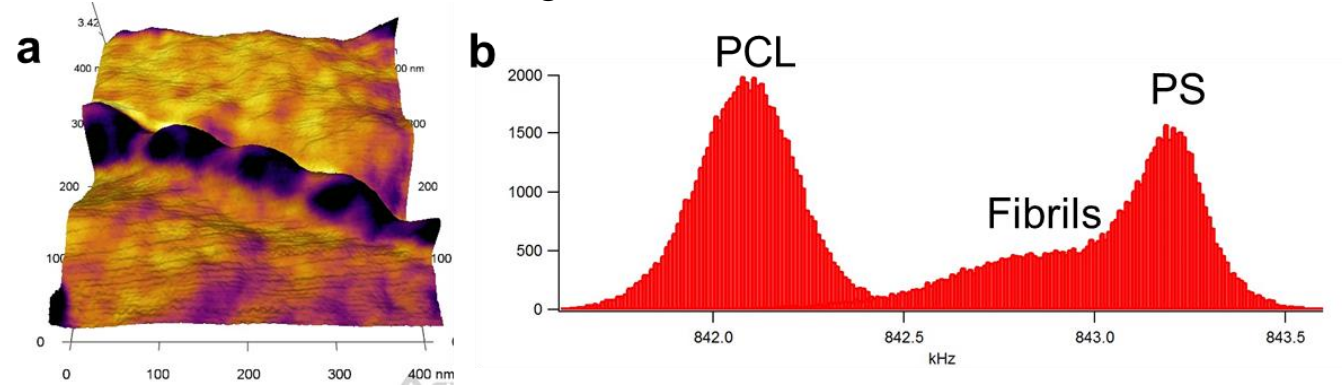

Figure 2. Frequency shift of the cantilever's second eigenmode during analysis of $\beta$-lactoglobulin fibrils as (a) an overlay onto the simultaneously measured topography on PS films and (b) a frequency distribution across several scan windows, identifying fibrils versus either PS or PCL background. 